\title{
The shortest modulation period Blazhko RR Lyrae star: SS Cnc
}

\author{
J. Jurcsik, B. Szeidl, and Á. Sódor, \\ Konkoly Observatory, P.O. Box 67, H-1525 Budapest, Hungary, \\ jurcsik@konkoly.hu \\ I. Dékány, Zs. Hurta, K. Posztobányi, and K. Vida, \\ Eötvös Loránd University, Dept. of Astronomy, P.O. Box 32, H-1518 Budapest, Hungary \\ M. Váradi \\ Konkoly Observatory, P.O. Box 67, H-1525 Budapest, Hungary \\ A. Szing \\ Dept. of Experimental Physics and Astronomical Observatory, University of Szeged, \\ Hungary
}

\begin{abstract}
Extended $B V(R I)_{C}$ CCD observations of SS Cnc, a short period RRab star are presented. Nearly 1400 data points in each band have been obtained spanning over 79 days during the spring of 2005. The star exhibits light curve modulation, the so called Blazhko effect with small amplitude ( $B$ maximum brightness varies $0.1 \mathrm{mag})$ and with the shortest modulation period (5.309 d) ever observed. In the Fourier spectrum of the $V$ light curve the pulsation frequency components are detected up to the 24th harmonic order, and modulation side lobe frequencies with significantly asymmetric amplitudes are seen up to the 15th and 9th orders for the lower and higher frequency components, respectively. Detailed comparison of the modulation behavior of SS Cnc and RR Gem, the two recently discovered small amplitude, short modulation period Blazhko stars is presented. The modulation frequency $\left(f_{m}\right)$ appears in the Fourier spectrum of both stars with similar amplitude. We also demonstrate that the modulation frequencies have basically different properties as the pulsation and modulation side lobe frequencies have, indicating that the physics behind these frequency components are not the same. The discovery of small amplitude modulations of RRab stars cautions that the large photometric surveys (MACHO, OGLE) may seriously underestimate the number of modulated RR Lyrae stars.
\end{abstract}

Subject headings: Stars: individual: SS Cnc, - Stars: variables: RR Lyr - Stars: oscillations - Stars: horizontal-branch - Techniques: photometric 


\section{Introduction}

RR Lyrae stars are of great astrophysical importance in many respects. They are standard candles of distance estimations, test objects of stellar evolution and pulsation model calculations. The physics governing their pulsation behavior is thought to be well understood, but there are still some puzzling properties of these objects as well. The most troublesome is the so called Blazhko effect, the light curve modulation of some fraction of RR Lyrae stars. Smith (1995) gives an overall description of the phenomenon, and for a recent summary of the problems of theoretical explanations see Dziembowski \& Mizerski (2004). First connections between some properties of the pulsation and the modulation have been established in Jurcsik et al. (2005b) and Jurcsik et al. (2005c).

The Blazhko phenomenon has been known for about a century. Most of the known galactic field RRab stars showing light curve modulation were discovered from photographic observations during the first part of the last century. Though the advent of the photoelectric, and in the last decades of the 20. century, the CCD era has led to spectacular increase of the photometric precision, there is still a lack of extended high accuracy survey of the modulation properties of the galactic field RR Lyrae stars in the Solar neighborhood.

The statistics of the occurrence frequency of the phenomenon are contradictory, e.g. Moskalik \& Poretti (2003) found 35\% and 5\% incidence rate for fundamental and first overtone variables in the Galactic Bulge, while Alcock et al. (2003) and Nagy \& Kovács (2006) detected $11.9 \%$ and $7.5 \%$ of the RR Lyrae stars to show modulation properties in the LMC, respectively. Because of the differences in the accuracy of the surveys, the photometric wavebands used, the extension of the datasets, and the analyzing methods applied, exact comparison of the different statistics is, however, questionable.

The recent discovery of the very small amplitude modulation of RR Geminorum (Jurcsik et al. 2005a) called our attention to that small amplitude modulations which have escaped notice in the previous photometric studies might be quite frequent. This possibility may override any previous statistics of the incidence rate of the modulation. In order to get a clear picture of the frequency of the modulation of galactic field RR Lyrae stars, we started

a systematic photometric survey at Konkoly Observatory to measure short period $(P<0.48$ d) fundamental mode RR Lyrae stars.

In this note we report the discovery of another RR Lyrae star, SS Cnc $\left(\alpha=08^{h} 06^{m} 25.56, \delta=\right.$ $+23^{\circ} 15^{\prime} 05^{\prime \prime} 8$, J2000), exhibiting small amplitude modulation. Previous photometric observations of SS Cnc are very sparse, altogether 24 accurate photometric data points have been published by different authors (Fitch et al. 1966; Sturch 1966; Epstein 1969). It is a metal rich $\mathrm{RR}$ Lyrae star, its $[\mathrm{Fe} / \mathrm{H}]=-0.03$ (see the metallicity compilation in Jurcsik \& Kovács 
1996).

\section{Observations and data reduction}

The observations were obtained with the automated $60 \mathrm{~cm}$ telescope of Konkoly Observatory, Svábhegy, Budapest equipped with a Wright 750x1100 CCD using $B V(R I)_{C}$ filters. The field of view was $17^{\prime} \times 24$ '. Data reduction was performed using standard $\operatorname{IRAF}^{1}$ packages.

Nearly 1400 frames were obtained in each passband on 35 nights during the spring of 2005 (between JD 2453388 and JD 2453467). The data were transformed to standard $B, V, R_{C}, I_{C}$ magnitudes by using observations of standard stars in M67 (Chevalier \& Ilovaisky 1991).

As no single comparison star with ideal properties (brightness, color, vicinity) has been found in the field we have measured relative magnitudes of SS Cnc to the mean magnitudes of three stars: GSC 01927-00504, GSC 01927-00744, and GSC 01927-00835.

The r.m.s. scatter of the averaged magnitude differences between these three stars is $0.008,0.009,0.009$, and $0.009 \mathrm{mag}$ in the $B, V, R_{C}, I_{C}$ colors, respectively.

Relative magnitudes of SS Cnc are available in machine readable format at the http:/www.konkoly.hu/2 web address.

\section{The shortest modulation period, 5.309 day, ever observed}

The observed pulsation light curve of SS Cnc (see Fig. 1) shows larger scatter than observational inaccuracies could explain. Fitting the light curve only with the pulsation frequency and its harmonics the r.m.s. scatter of the residual data is 2-3 times larger than expected, $0.028,0.022,0.017$ and 0.014 mag in the $B V R_{C} I_{C}$ colors, respectively.

The brightness of maximum light varied from night to night, indicating Blazhko modulation of the light curve. Detailed Fourier analysis of the data has revealed that this is indeed the case, but the modulation period of SS Cnc is unexpectedly short, only $5.309 \mathrm{~d}$.

\footnotetext{
${ }^{1}$ IRAF is distributed by the National Optical Astronomy Observatories, which are operated by the Association of Universities for Research in Astronomy, Inc., under cooperative agreement with the National Science Foundation.
} 
In Fig. $2 \Delta B$ observations are plotted against HJD and the phase of the detected short period modulation.

The pulsation and modulation periods (mean values obtained for the $B V R_{C} I_{C}$ data) have been determined from Fourier analysis, using the facilities of the program package MUFRAN (Kolláth 1990). We have detected 24 harmonics of the pulsation frequency $\left(f_{0}=\right.$ $2.722296 \mathrm{c} / \mathrm{d}$ ) in the Fourier spectrum of the $V$ light curve, and modulation peaks up to the 15 th and 9th orders at smaller and larger frequency values than the pulsation components, respectively (see Fig. 3). The positions of the side lobe modulation frequencies $\left(k f_{0} \pm f_{m}\right)$ correspond to $f_{m}=0.18835 \mathrm{c} / \mathrm{d}$, i.e., $5.309 \mathrm{~d}$ period of the modulation. The following ephemerides give maximum pulsation light and maximum amplitude timings:

$$
\begin{aligned}
& \text { HJD } T_{\max }=2453464.459+0.367337 E_{\text {pulsation }}, \\
& \text { HJD } T_{\text {Amax }}=2453410.6+5.309 E_{\text {Blazhko }} .
\end{aligned}
$$

The amplitudes $\left(A_{V}\right)$ and phases $\left(\phi_{V}\right)$ of the detected frequencies of the $V$ light curve and $A_{B} / A_{V}, A_{V} / A_{R}$ and $A_{V} / A_{I}$ amplitude ratios and $\phi_{B}-\phi_{V}, \phi_{V}-\phi_{R}$ and $\phi_{V}-\phi_{I}$ phase differences are listed in Table 1 . The phases correspond to sine term solutions with initial epoch value: $T_{0}=2453388.000$.

The amplitudes of the modulation side lobe frequencies $\left(k f_{0} \pm f_{m}\right)$ are strongly asymmetric, the smaller frequency components $\left(k f_{0}-f_{m}\right)$ have significantly larger amplitudes than the longer frequency ones $\left(k f_{0}+f_{m}\right)$. With the exception of the second order, the amplitudes of the $k f_{0}+f_{m}$ components do not exceed 0.005 mag in $V$ band. This order of magnitude signal definitely escapes detection in the case of less extended datasets and/or worse $\mathrm{S} / \mathrm{N}$ ratio.

This result warns that the classification scheme of the modulation which is based on that the modulation side lobe frequencies are present only at one side of the pulsation components ( $\nu 1$ variables) or at both sides (Blazhko variables) might be seriously affected by data incompleteness and $\mathrm{S} / \mathrm{N}$ properties. A similar conclusion has already been drawn in Jurcsik et al. (2005a) based on that the Fourier spectra of the two halves of the extended dataset of RR Gem show substantially different character of the modulation. According to the data sampling and accuracy of the existing photometric surveys, SS Cnc would have been classified as a $\nu 1$ variable if its modulation had been detected at all. 


\section{Comparison of the modulation properties of the two small modulation amplitude Blazhko stars, SS Cnc and RR Gem}

Our multicolor photometric observations of SS Cnc and RR Gem (Jurcsik et al. 2005a) are extended enough to study and to compare the modulation characteristics of these stars in detail. Though both stars exhibit modulation with extremely short period and small amplitude their modulation properties are different in many respects.

Removing the mean pulsation light curves from the $V$ data the residual light curves against the pulsation phases are shown in Fig. 4 for both stars. These plots indicate only slight similarity, while the residual plot of RR Gem is symmetrical to phase 0.5 (which corresponds to the mid of the rising branch in both plots), there is no symmetry in the residual data of SS Cnc. The only similarity of the two plots is that large modulation amplitude is restricted to a narrow phase interval in the vicinity of phase 0.5. Similar residual phase plot of DR And, a large modulation amplitude Blazhko star was shown in Lee \& Schmidt (2001). The modulation is the largest on the ascending branch of DR And, too, but increased modulation extends to a wider, 0.4 pulsation phase interval.

The residual light curves of the Blazhko variables give some impression about the character of the modulation. In case of pure amplitude modulation the residual is the largest at around minimum and maximum light and it is the smallest at around mean brightness values. On the contrary, pure phase modulation results in large residual amplitude at around mid brightnesses and small residual amplitudes around the extrema. Based on these properties Fig. 4 indicates that in SS Cnc phase modulation does also occur, while in RR Gem the amplitude modulation is dominant.

The data coverage enabled us to analyze the displacement of the extrema and the shape of the light curves in the different phases of the Blazhko cycle separately. In order to do this, we have divided the $V$ dataset of the two stars into ten-ten subsets, each containing data of a full pulsation light curve but in different phase intervals of the Blazhko cycle. (In SS Cnc there is a 0.2 pulsation phase gap in one of these light curves which was filled by linear interpolation of the data subsets of the preceding and following Blazhko phases.)

The variations in the heights and phases of the maxima in 10 different Blazhko phases are shown in Fig. 5 for the two stars. From these plots it is evident that in both stars both amplitude and phase modulation occur, but, indeed, in RR Gem amplitude modulation is dominant. Maxima timings precede the expected value the most at around the largest amplitude phase of the Blazhko cycle both in SS Cnc and in RR Gem.

In Fig. 6 the residual light curves of SS Cnc in the 10 different phases of the Blazhko cycle are separately plotted. Again, it is evident that large amplitude modulation occurs 
in a restricted phase interval around phase 0.5. This concentration of the modulation to a narrow phase interval of the pulsation results in enhanced amplitudes of the higher order modulation components. Fig. 7 compares the amplitude decrease of the pulsation and modulation frequency components with increasing harmonic orders of the two stars. In contrast to the exponential amplitude decrease of the pulsation components, the amplitudes of the modulation frequencies decrease less steeply. Another interesting feature is that, though the $k f+f_{m}$ and $k f-f_{m}$ components have the same amplitudes in RR Gem and are significantly different in SS Cnc, the mean values of the amplitudes at a given order are very similar in the two stars. It is also important to note that $f_{m}$ appears with commensurable amplitude like the first modulation frequencies in both cases.

The Fourier parameters of the pulsation light curves of the 10 data subsets for SS Cnc and RR Gem are plotted in Fig. 8. RR Gem shows the largest variation in the Fourier amplitudes and amplitude ratios $\left(R_{k 1}=A_{k} / A_{1}\right)$ during the Blazhko cycle, while most of the Fourier parameters of SS Cnc vary significantly. These properties reflect also that amplitude modulation is dominant in RR Gem, while in SS Cnc both amplitude and phase modulation have significant amplitudes. An interesting feature of SS Cnc is that the variation in the amplitude ratios and the phase differences $\left(\phi_{k 1}=\phi_{k}-k \phi_{1}\right)$ are not harmonized in phase. It is worth noting that the amplitude of the variation increases towards the higher order phase differences.

As both SS Cnc and RR Gem were used as calibrating objects of the light curvephysical parameter relations of fundamental mode RR Lyrae stars, we have also checked how the detected changes in the Fourier parameters influence the value of the derived physical parameters. The calculated $[\mathrm{Fe} / \mathrm{H}]$ values in the different Blazhko phases vary by about 0.2 according to the formula which involves the period and $\phi_{31}$ (Jurcsik \& Kovács 1996). In the calculated absolute brightness values (using the formula derived for $\operatorname{Set} A$ in Kovács \& Walker 2001) there are less than $0.02 \mathrm{mag}$ differences for both of the stars. These values have the same order of magnitudes if compared to the estimated accuracies of the formulae. We can thus conclude that small amplitude modulation of RRab stars probably does not influence severely the applicability of the light curve-physical parameter formulae.

It was already shown in Jurcsik et al. (2005a) that in contrast with theoretical expectations the amplitude ratios and the phase differences of the $k f_{0}+f_{m}$ and $k f_{0}-f_{m}$ modulation side lobe component pairs in the different colors and orders do not have the same value. The largest deviations of the four colors and the first five harmonic orders are 0.25 in the amplitude ratios and $30 \mathrm{deg}$ in the phase differences in RR Gem. These differences are substantially larger, 3.1 and $76 \mathrm{deg}$ in SS Cnc, respectively. There are slight, but significant differences between the results in the four colors of the same order, and unexpectedly large differences 
between the different order's data.

\subsection{Color changes}

The intensity mean color changes during the Blazhko cycle indicate some tens of degrees mean effective temperature changes of RR Gem (Jurcsik et al. 2005a). The star seems to be slightly cooler in its smaller amplitude state than when its pulsation amplitude is larger. Fig. 9 shows the magnitude and intensity mean $V, B-V$ and $V-I_{C}$ magnitudes and colors in the different phases of the Blazhko cycle of SS Cnc. The means are defined as the zero point term of the Fourier fits of the full pulsation light curves in each Blazhko phase interval. These values have been determined for the $B, V, R_{C}, I_{C}$ light curves in the different Blazhko phases, and the mean colors correspond to the differences of the mean magnitudes in the different wavelength bands. The accuracy of the mean magnitudes is estimated to be around 0.001-0.002 mag.

Both the intensity and magnitude mean $V$ brightnesses of SS Cnc vary during the Blazhko cycle. Their amplitudes are about 0.012-0.015 mag. The brightest and faintest states occur about 0.1-0.3 Blazhko phase after the phases of maximum and minimum amplitudes, respectively. The $\langle B\rangle-\langle V\rangle$ color index does not show systematic variation but its $0.005-$ $0.007 \mathrm{mag}$ scatter is larger than expected from the estimated accuracy of the data. The $\langle V\rangle-\langle I\rangle$ shows less than 0.01 mag amplitude variation in line with the changes of the mean $V$ brightness.

\section{The discrepant properties of $f_{m}$}

It was a matter of debate for long whether the modulation frequency has observable amplitude in Blazhko stars at all. Kovács (1995) and Nagy (1998) detected 0.011 and 0.005 mag amplitudes of the modulation frequency in the $B$ data of the two large modulation amplitude Blazhko stars, RV UMa and RS Boo. During the modulation period the average brightness varies with an overall amplitude of $0.006 \mathrm{mag}$ in 731 Blazhko variables in the LMC (Alcock et al. 2003). We have detected 0.006 and 0.008 mag $B$ amplitudes of the modulation frequency of RR Gem and SS Cnc, respectively. These results indicate that it is a general property of Blazhko stars that $f_{m}$ have similar amplitudes of the order of or less than 0.01 mag, independently of the amplitude and other characteristics of the modulation. This is in contrast with the large variety of the amplitudes of the $k f_{0} \pm f_{m}$ modulation components, which exceed even $0.1 \mathrm{mag}$ in some cases (see e.g. Smith et al. $(1994,1999))$. 
Another peculiarity of the $f_{m}$ frequency component is summarized in Table 2. This table lists the amplitude ratios and phase differences of the different color curve solutions for the modulation $\left(f_{m}\right)$, and for the mean values of the pulsation $\left(k f_{0}\right)$, and modulation 'side lobe' frequencies $\left(k f_{0} \pm f_{m}\right)$ for SS Cnc and RR Gem. There are no differences in the mean values of the amplitude ratios and phase differences of the pulsation and modulation side lobe frequency values of the two stars, they have the same values within the range of the scatter of the values for the different harmonic orders of the same star. The amplitude ratios detected for $f_{m}$ are, however, significantly different from these values. Towards redder colors the amplitudes of $f_{m}$ are much smaller for SS Cnc and much larger for RR Gem than detected for all the other frequency components. The $A_{B} / A_{V}$ amplitude ratios of $f_{m}$ are the less deviant, but for SS Cnc it is definitely smaller than the $A_{B} / A_{V}$ amplitude ratios of the other frequency components.

It is a natural expectation that the physics of the light variation determine the amplitude ratios in different colors. Therefore, the different color dependence of the amplitudes of $f_{m}$ and the similarity of the amplitude ratios of the pulsation and modulation side lobe frequencies indicate that $f_{m}$ has basically different physical origin than the radial mode pulsation has, while the side lobe frequencies share somehow the properties of the pulsation. If nonlinear interaction between a millimagnitude amplitude signal $\left(f_{m}\right)$ and the two orders of magnitude larger amplitude pulsation calls forth to the appearance of the side lobe frequencies, then the properties of the combination frequencies would more probably inherit the properties of the dominant pulsation frequencies than those of the small amplitude signal.

This result if strengthened by detailed observations of other Blazhko stars may lead to the conclusion that the real physically reasonable frequency of the modulation is in fact $f_{m}$. Any interpretation of the modulation which is connected to nonradial mode frequencies excited in the vicinity of the radial mode pulsation frequency may have difficulties to explain the discrepant behavior of $f_{m}$, which might question the validity of the identification of any of the side lobe frequencies with a nonradial mode frequency.

\section{Conclusions}

Based on our photometric investigation of SS Cnc, and the previous study of RR Gem we add the following empirical facts to our knowledge of the modulation properties of RRab stars.

- Small amplitude modulations with Fourier amplitudes of the modulation frequency components of the order of millimagnitudes can be detected in RRab stars. 
- The amplitude decrease of the modulation components towards higher harmonic orders is significantly less steep than the amplitude decrease of the harmonic components of the pulsation.

- The changes of the Fourier parameters of the pulsation light curve in the different Blazhko phases are star by star different.

- Based on the variations of the mean colors during the Blazhko cycle the mean effective temperatures of the stars vary by less than $\sim 50 \mathrm{~K}$.

- The color dependences of the amplitudes and phases of the pulsation and modulation side lobe frequencies are the same in the different harmonic orders for the two small modulation amplitude stars, but the modulation frequency itself $\left(f_{m}\right)$ shows significantly different color behavior.

These items, though still do not solve the mystery of the Blazhko phenomenon add important contributions to our knowledge of the modulation properties of RRab stars, which have to be explained by any valid physical description. As a concluding remark we also emphasize that the discovery of small amplitude modulations questions the validity of any previous statistics on the occurrence of the modulation. Based on accurate, detailed photometric data we also think that the current classification of the modulation into $\nu_{1}$ and Blazhko types according to the amplitudes of the modulation side lobe frequencies is just an artifact of noisy data and bad data sampling.

Up to now 4 galactic field Blazhko stars are known with modulation periods shorter than 20 days: SS Cnc, RR Gem, AH Cam (Smith et al. 1994), and CZ Lac (Jurcsik et al. 2006). It was shown in Jurcsik et al. (2005b) that the modulation period only of the short pulsation period RRab stars can be anomalously short. A common feature of these stars is that they are all metal rich, with metallicity close to the solar value. However, metallicity does not have a definite effect on the period of the modulation. Metal rich, short pulsation period Blazhko stars with long modulation periods do also exist, e.g., the modulation period of RS Boo is 530 days $\left(P_{\text {puls }}=0.38 \mathrm{~d},[\mathrm{Fe} / \mathrm{H}]=-0.2\right)$.

An important result of our investigations is the demonstration of the discrepant behavior of the modulation frequency, $f_{m}$ itself. The invariance of its amplitude in Blazhko stars and the discrepant color dependence of the amplitudes of this component indicate that the triggering mechanism of the light variations with the modulation period is basically different than that of the radial mode pulsation. The amplitudes of the side lobe modulation frequencies have the same color dependence as the pulsation frequencies have suggesting that these components are physically better connected to the radial mode. 
The explanations of the Blazhko phenomenon involving nonradial modes do not give a clear picture yet. Van Hoolst et al. (1998) have found many unstable low degree $(l=1,2)$ nonradial $g$-modes of high radial order $(n>80)$ in RR Lyrae models. These modes have frequencies in the vicinity of the frequencies of the observed radial modes. However, there is no indication in the literature, that the low frequency domain $\left(f_{m}<0.15 \mathrm{c} / \mathrm{d}\right)$ would have been searched for unstable nonradial modes as well. The only model which explains the existence of the side lobe frequencies and is more or less in accordance with observations identifies these frequencies with rotation induced $m= \pm 1$ pairs (Nowakowski \& Dziembowski 2001). This model cannot explain, however, the observed high amplitudes and the strong asymmetry of the triplets (Dziembowski \& Mizerski 2004).

The discrepant behaviour of the light variations with the modulation frequencies urges theoretical investigations focusing on the possibility that short frequency nonradial modes in RR Lyraes stars similarly to $\gamma$ Dor variables, may also exist.

We would like to thank the referee, Horace Smith his useful comments which helped to improve the lucidity of the paper. The financial support of OTKA grants T-043504, T-046207 and T-048961 is acknowledged.

\section{REFERENCES}

Alcock, C., et al. 2003, ApJ, 598, 597

Chevalier, C. \& Ilovaisky, S. A. 1991, A\&ASupplement Series, 90, 225

Dziembowski, W. A. \& Mizerski, T. 2004, Acta Astronomica, 54, 363

Epstein, I. 1969, AJ, 74, 1131

Fitch, W. S., Wiśniewski, W. Z., Johnson, H. L. 1966, Comm. Lunar and Planet. Lab. Vol. 5, No. 71

Jurcsik, J. \& Kovács, G. 1996, A\&A312, 111

Jurcsik, J., Sódor, Á., Váradi, M., Szeidl, B. et al. 2005a, A\&A, 430, 1049

Jurcsik, J., Szeidl, B., Nagy, A. \& Sódor, Á. 2005b, Acta Astronomica, 55, 303

Jurcsik, J., Sódor, Á., \& Váradi, M. 2005c, IBVS, No. 5666

Jurcsik, J. et al. 2006, in preparation 
Kolláth, Z. 1990, Occ. Techn. Notes Konkoly Obs., No. 1, http://www.konkoly.hu/staff/kollath/mufran.html

Kovács, G. 1995, A\&A, 295, 693

Kovács, G. \& Walker, A. 2001, A\&A, 371, 579

Lee, K. M. \& Schmidt, E. G. 2001, PASP, 113, 1140

Moskalik, P. \& Poretti, E. 2003, A\&A, 398, 213

Nagy, A. 1998, A\&A, 339, 440

Nagy, A. \& Kovács, G. 2006, A\&A, accepted

Nowakowski,R. \& Dziembowski, W. 2001, Acta Astronomica, 51, 5

Smith, H. A. 1995, RR Lyrae Stars (Cambridge Univ. Press)

Smith, H. A., Barnett, M., Silbermann, N. A. \& Gay, P. 1999, AJ, 118, 572

Smith, H. A., Matthews, J., Lee, K. K., Williams, J., Silbermann, N. A. \& Bolte, M. 1994, AJ, 107, 679

Sturch, C. R. 1966 ApJ, 143, 774

Van Hoolst, T., Dziembowski, W. \& Kawaler, S. 1998, MNRAS, 297, 536 


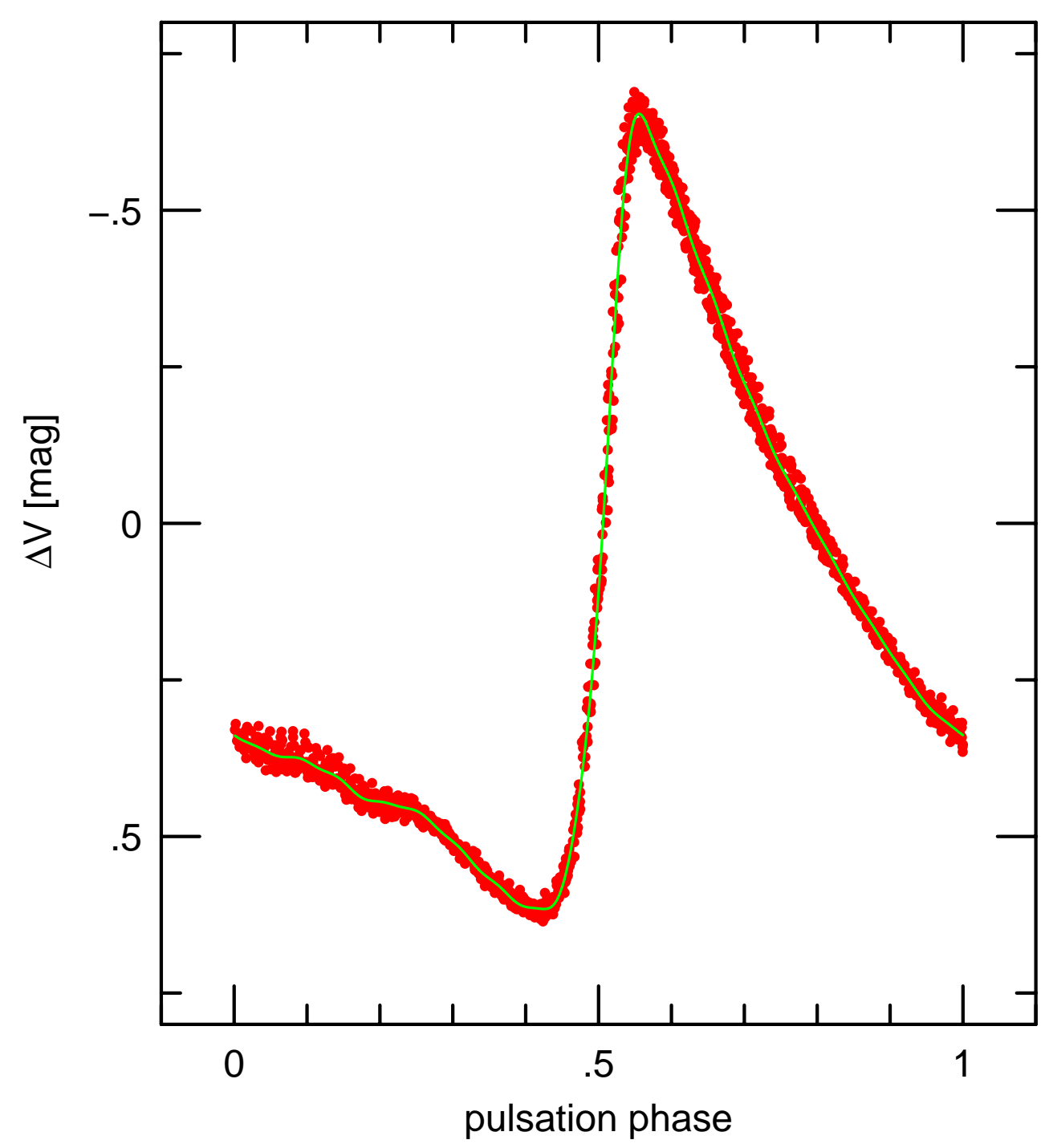

Fig. 1. $-\Delta V$ light curve of SS Cnc folded with the $0.367337 \mathrm{~d}$ pulsation period. The line corresponds to a fit with the pulsation frequency and its 24 harmonic components. The 0.05-0.10 mag width of the light curve is due to modulation with an extremely short, 5.309 $\mathrm{d}$ period, and of small amplitude. Phase 0.5 corresponds to the mid of the rising branch, where the measured magnitude equals to the intensity mean magnitude. 

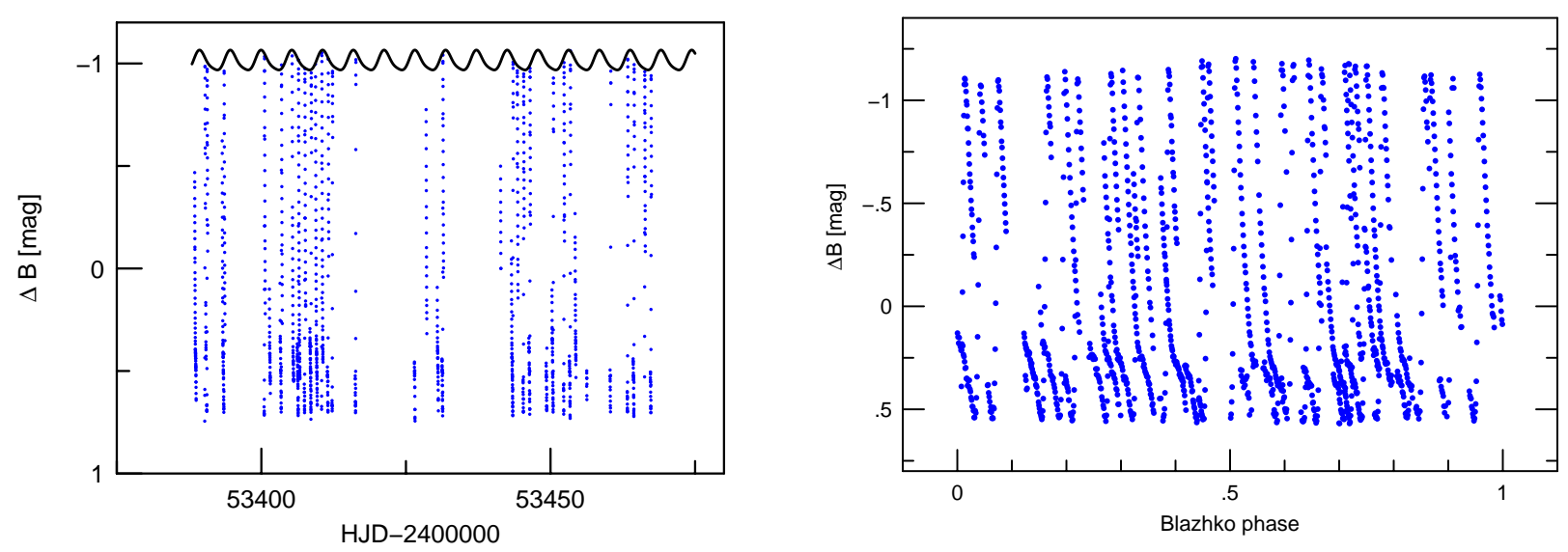

Fig. 2.- $\Delta B$ observations of SS Cnc (left panel) and data folded according to the phase of the detected $5.309 \mathrm{~d}$ modulation period (right panel). Due to the shortness of the pulsation period data cover maximum light in most of the nights of the observations. Therefore, the upper envelope of all the measurements shown in the left panel indicates clearly modulation with $5.309 \mathrm{~d}$ and total amplitude of $0.10 \mathrm{mag}$. The asymmetric shape of the envelope is a sign that beside amplitude modulation phase modulation is also present. 


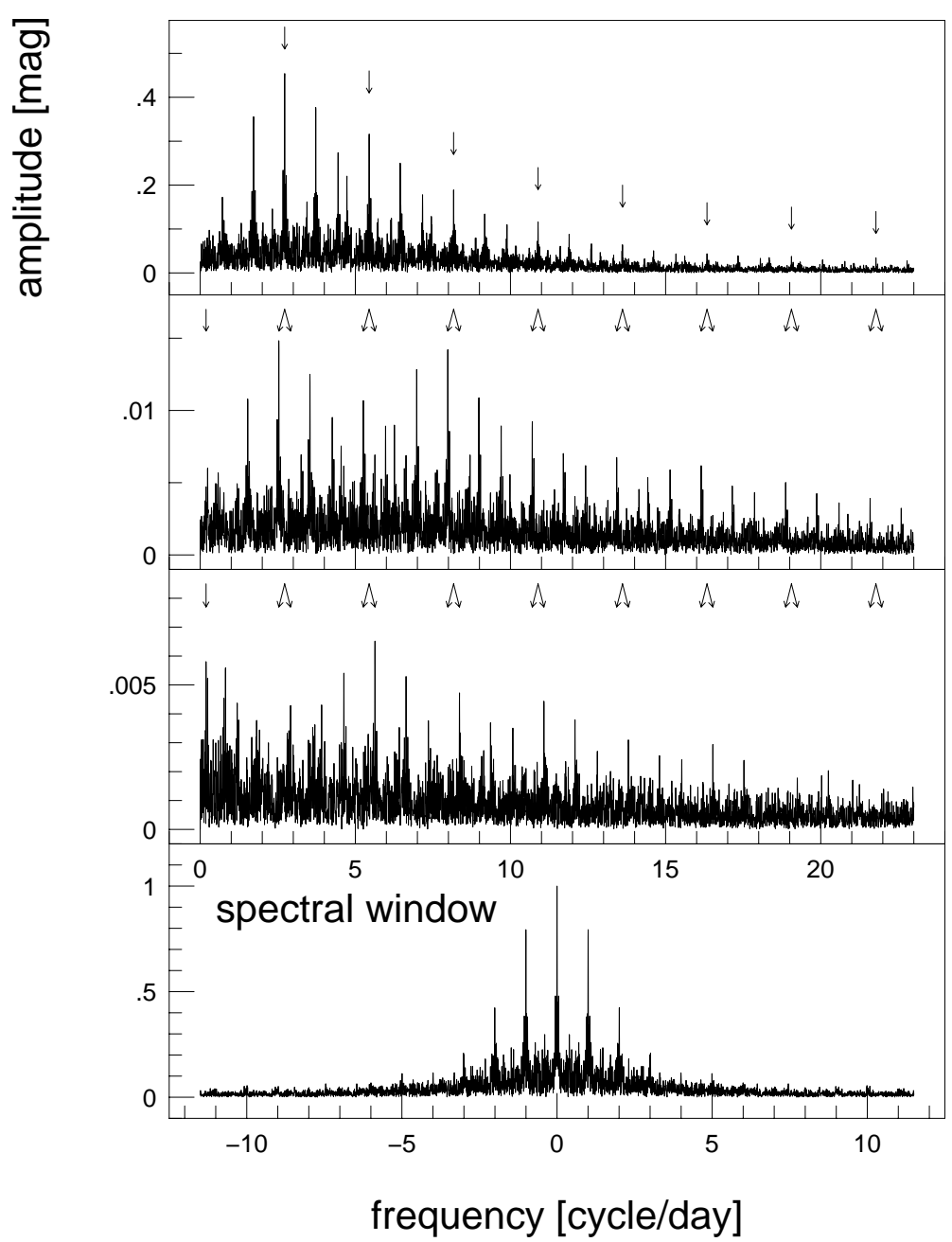

Fig. 3.- Fourier spectrum of the $V$ light curve of SS Cnc. In the top panel arrows point to the pulsation frequency and its harmonics $\left(k f_{0}\right)$. The other large amplitude peaks are alias frequencies of these components. The next panel shows the prewhitened spectrum after the removal of the signal of the radial mode pulsation. In this spectrum modulation frequency components appear at $0.18835 \mathrm{c} / \mathrm{d}$ shorter positions than the pulsation frequencies $\left(k f_{0}-f_{m}\right)$. Arrows show modulation frequency positions at the sides of equidistant triplets $\left(k f_{0} \pm f_{m}\right)$ appearing typically in the spectra of Blazhko stars. The larger frequency side lobe modulation components $\left(k f_{0}+f_{m}\right)$ can be detected only after the $k f_{0}-f_{m}$ components have been removed as shown in the next panel. It is apparent that the amplitudes of the modulation side lobe frequencies are strongly asymmetric, among the longer frequency components only $2 f_{0}+f_{m}$ has an amplitude larger than 0.005 mag. The separations of the modulation frequency components correspond to $5.309 \mathrm{~d}$ period of the modulation. Bottom panel shows the spectral window. 

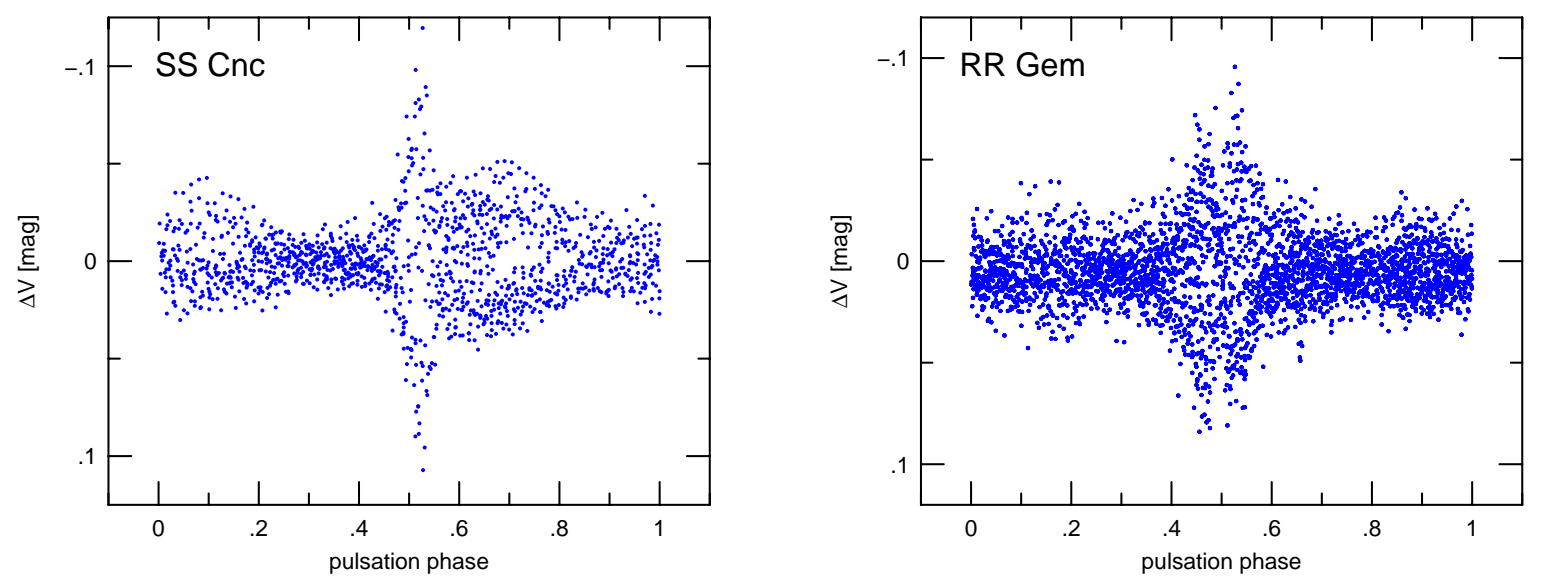

Fig. 4.- Residual light curves of SS Cnc (left) and RR Gem (right) after the removal of the mean light variation according to a Fourier fit to the data with the pulsation frequency and its harmonics. In both plots phase 0.5 corresponds to the mid of the rising branch. In SS Cnc some hundredths of magnitude modulation can be seen in most phases of the pulsation, except between the phases $0.25-0.45$ which falls into the 0.2 phase interval preceding minimum light (see Fig. 1). The amplitude of the modulation is the largest on the rising branch, in the phase interval 0.47-0.55. The residual light curve of RR Gem is different. Its symmetry to phase 0.5 is evident, enhanced modulation occurs in two lobes in 0.1 phase intervals preceding and following phase 0.5. In SS Cnc the left side lobe of the residual curve seems to be missing. 

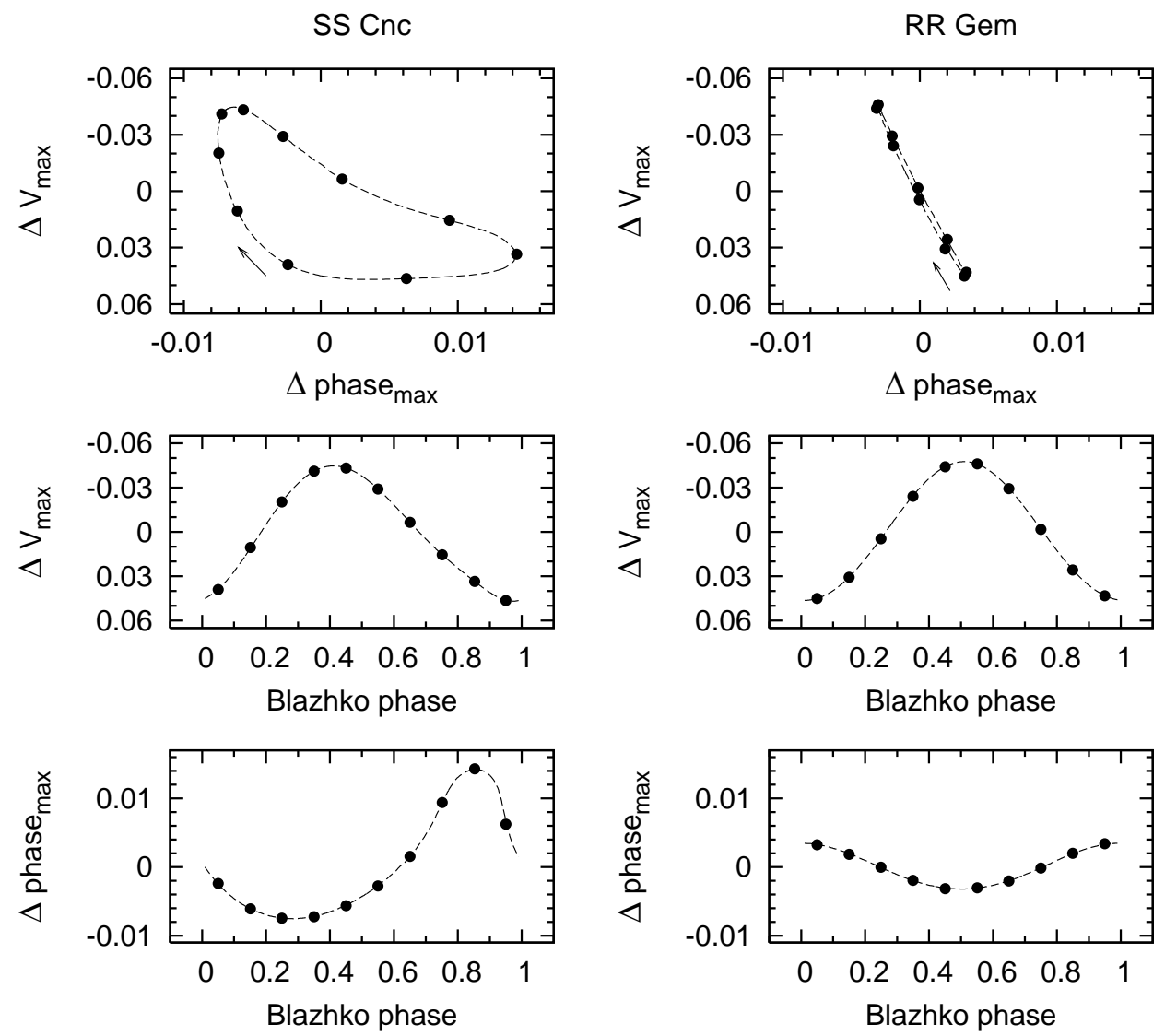

Fig. 5.- Variations in the heights and phases of maxima of SS Cnc and RR Gem during the Blazhko cycle are plotted. $\Delta V_{\max }$ and $\Delta$ phase $_{\max }$ denote $V_{\max }-\left\langle V_{\max }\right\rangle[\mathrm{mag}]$ and phase $_{\max }-\left\langle\right.$ phase $\left._{\max }\right\rangle[\mathrm{d}]$, respectively. Both stars exhibit amplitude and phase modulations, but in RR Gem the amplitude modulation is by far dominant. In the top figures arrows show the amplitude and phase variations between the first two Blazhko phases, the progression during the Blazhko cycle is clockwise in both stars. 


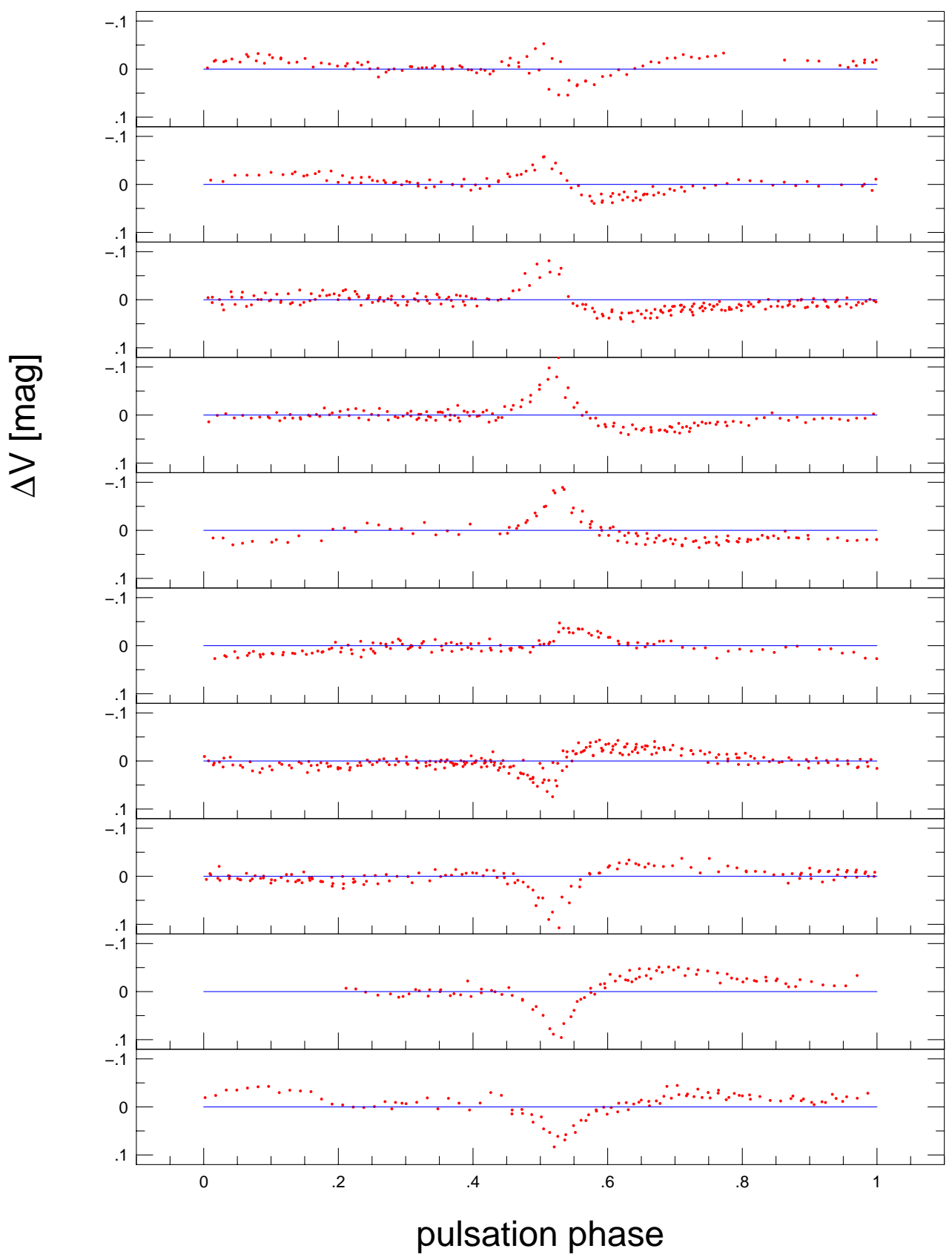

Fig. 6.- Residual light curves of SS Cnc belonging to 0.1 Blazhko phase intervals are plotted separately. 

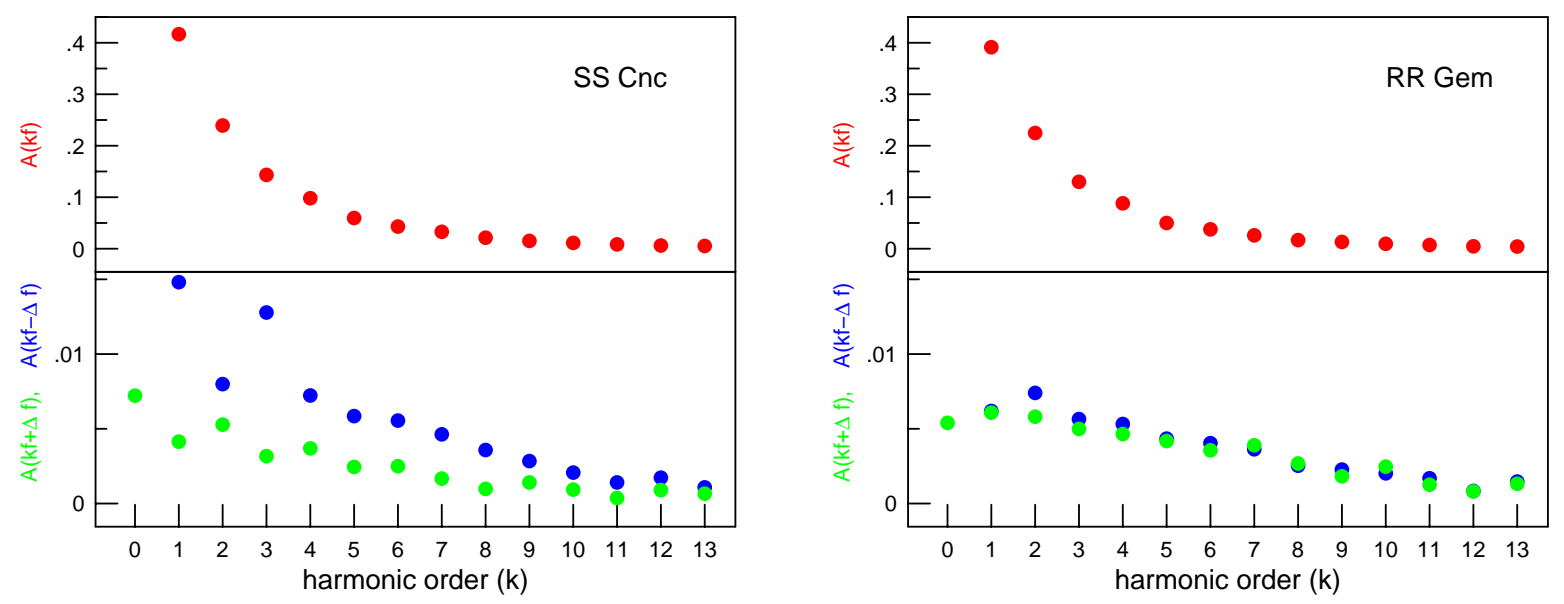

Fig. 7.- Fourier amplitudes of the pulsation and modulation frequencies for SS Cnc and RR Gem in $V$ band. In both cases the amplitudes of the pulsation frequencies decrease exponentially towards higher orders, while the amplitudes of the modulation frequencies decrease roughly linearly with some irregularity. The $k f_{0}-f_{m}$ and $k f_{0}+f_{m}$ modulation components of SS Cnc have significantly different, asymmetric amplitudes while for RR Gem they have similar amplitudes. It is an interesting feature, that in both figures the amplitudes of the second order modulation components show different character as the first and third order ones, it is more symmetric in SS Cnc and more asymmetric in RR Gem than the amplitudes of the neighboring order modulation frequencies. 

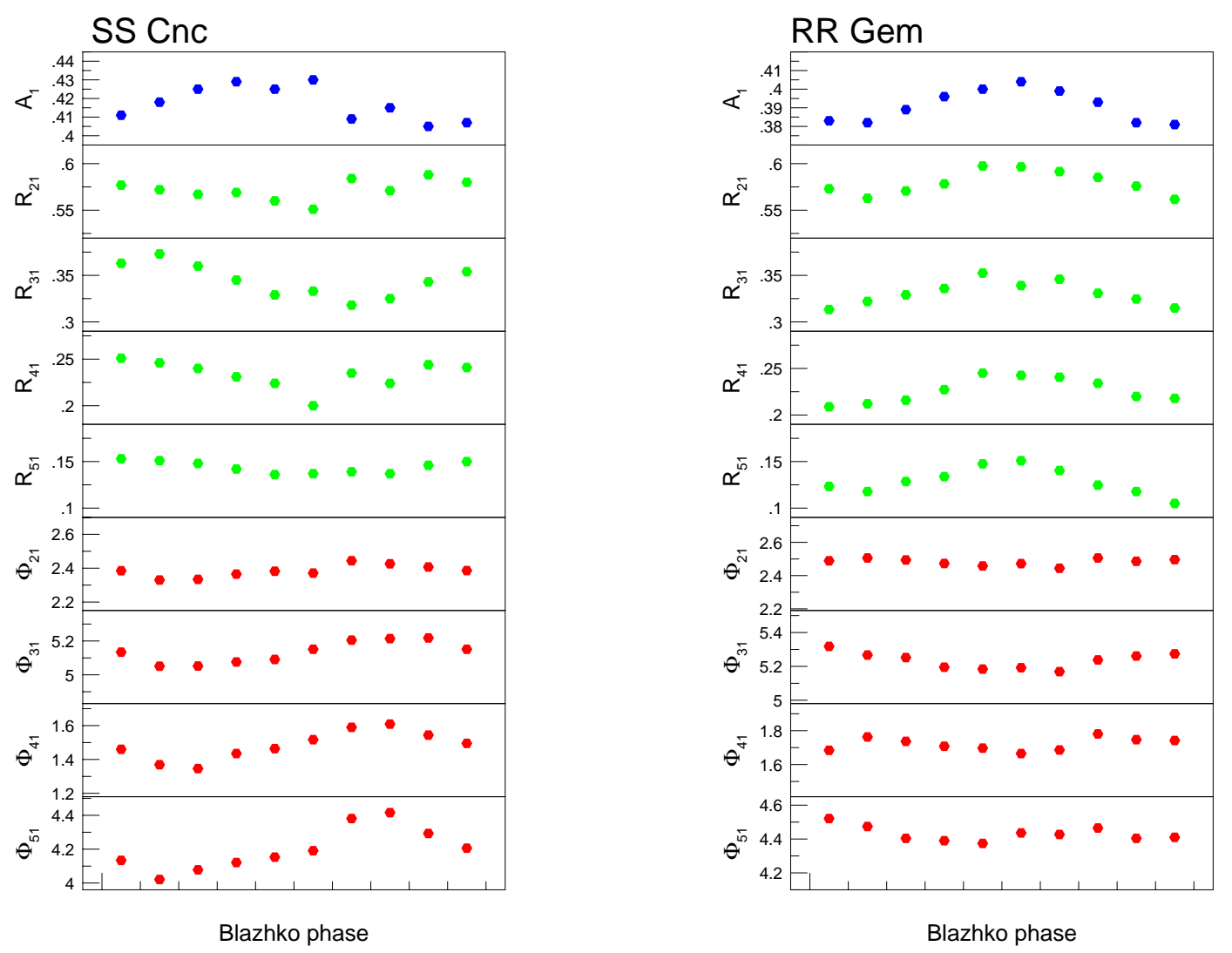

Fig. 8.- Fourier parameters of the $V$ light curves in the different phases of the Blazhko cycle of SS Cnc, and for comparison, of the other small amplitude, short modulation period Blazhko star, RR Gem are plotted. A1 varies 0.02-0.03 mag during the Blazhko cycle in both of the plots (top panels), while the amplitude ratios $\left(R_{k 1}, 2 .-5\right.$. panels) and phase differences ( $\Phi_{k 1}, 6 .-9$. panels) show different patterns in the two stars. In SS Cnc the $R_{31}$ and $R_{41}$ amplitude ratios indicate variations which is not in phase with the variation of $A 1$. Parallel changes of the phase differences can be seen with increasing amplitudes towards the higher orders: it is less than $0.1 \mathrm{rad}$ in $\Phi_{21}$ but reaches $0.4 \mathrm{rad}$ in $\Phi_{51}$. This complex behavior of the changes in the Fourier parameters reflects the compound, phase and amplitude modulation character of the Blazhko variation of SS Cnc. On the contrary, RR Gem exhibits much more simple variations of its Fourier parameters. Only the amplitudes show significant changes, the amplitude ratios show similar amplitude, parallel variations with $A 1$. This may be the consequence of the dominance of its amplitude modulation and the symmetric behaviour of its amplitude and phase modulations as shown in Fig. 5. 

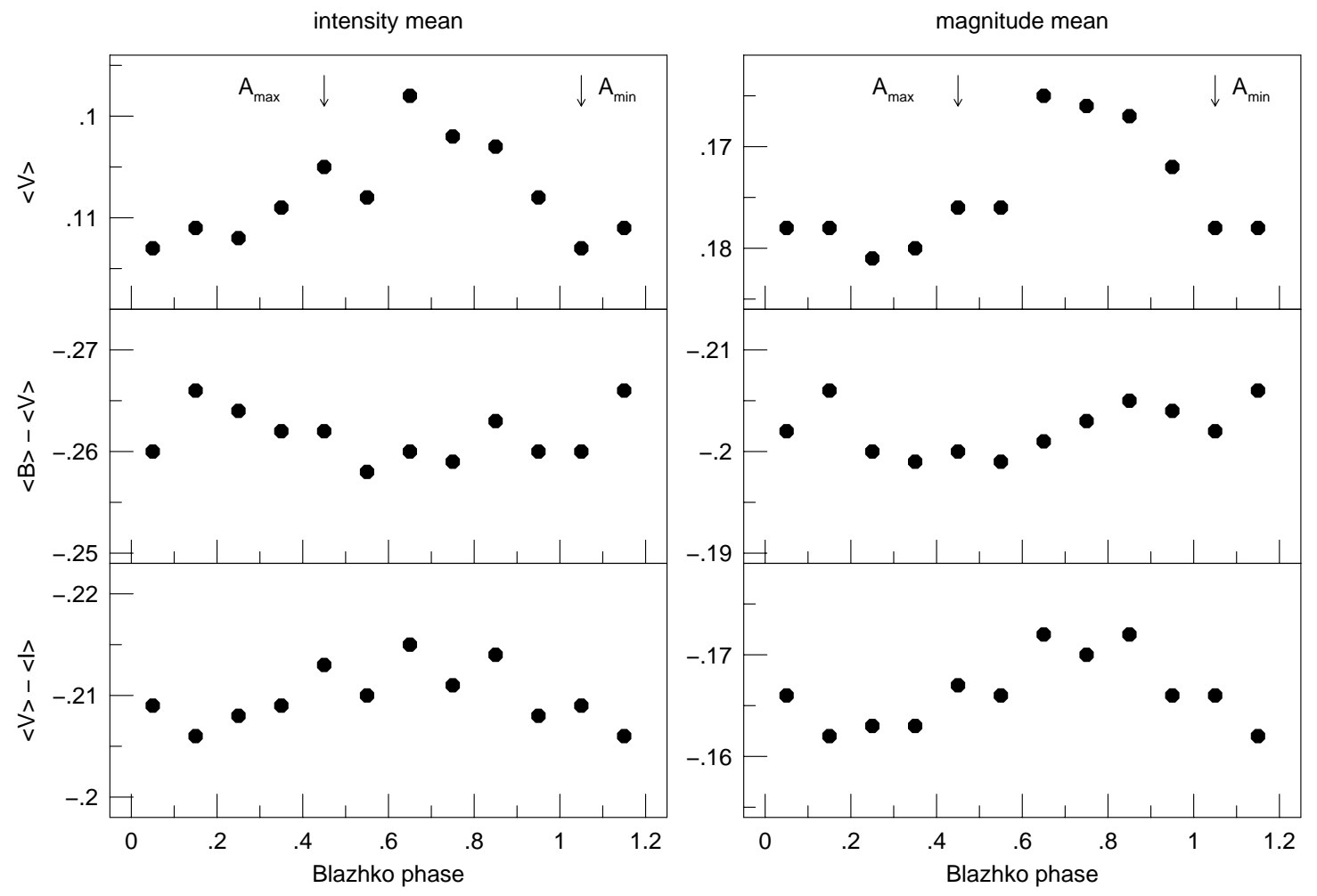

Fig. 9.- Intensity and magnitude mean $\langle V\rangle$ brightness and $\langle B\rangle-\langle V\rangle$ and $\langle V\rangle-\langle I\rangle$ color changes during the Blazhko cycle of SS Cnc. Both intensity and magnitude mean $\langle V\rangle$ values indicate slight, $\sim 0.01$ mag changes with minimum and maximum brightnesses preceding by 0.1-0.3 in phase the minimum and maximum amplitude Blazhko phases. The color changes are even smaller than the detected changes in the mean brightnesses, they are of the order of millimagnitudes. The $\langle V\rangle-\langle I\rangle$ mean colors show slight parallel changes with the mean brightness variations, while the $\langle B\rangle-\langle V\rangle$ values do not show any definite trend. 
Table 1. Fourier amplitudes (amplitude ratios) and phases (phase differences) of the detected frequencies in the $B V R_{C} I_{C}$ colors of SS Cnc

\begin{tabular}{|c|c|c|c|c|c|c|c|c|c|}
\hline & frequency c/d & $A_{V} \mathrm{mag}$ & $\phi_{V}$ deg. & $A_{B} / A_{V}$ & $\phi_{B}-\phi_{V}$ & $A_{V} / A_{R}$ & $\phi_{V}-\phi_{R}$ & $A_{V} / A_{I}$ & $\phi_{V}-\phi_{I}$ \\
\hline$f_{m}$ & 0.188350 & 0.00736 & 3.5 & 1.147 & -11.0 & 1.543 & 2.9 & 2.462 & 6.3 \\
\hline$f_{0}-f_{m}$ & 2.533946 & 0.01491 & 332.3 & 1.298 & -1.6 & 1.289 & 2.1 & 1.734 & -10.4 \\
\hline$f_{0}$ & 2.722296 & 0.41668 & 169.3 & 1.370 & 2.5 & 1.273 & 3.3 & 1.678 & 8.9 \\
\hline$f_{0}+f_{m}$ & 2.910646 & 0.00422 & 205.6 & 1.322 & -5.5 & 1.311 & 2.2 & 1.737 & -20.1 \\
\hline $2 f_{0}-f_{m}$ & 5.256242 & 0.00796 & 257.0 & 1.180 & -6.8 & 1.259 & 4.2 & 1.502 & -1.0 \\
\hline $2 f_{0}$ & 5.444592 & 0.23932 & 115.5 & 1.342 & 0.4 & 1.250 & 0.6 & 1.627 & 2.1 \\
\hline $2 f_{0}+f_{m}$ & 5.632942 & 0.00546 & 172.9 & 1.158 & -5.1 & 1.273 & 0.4 & 1.722 & -2.9 \\
\hline $3 f_{0}-f_{m}$ & 7.978538 & 0.01290 & 191.9 & 1.384 & 1.7 & 1.287 & -4.7 & 1.746 & 2.7 \\
\hline $3 f_{0}$ & 8.166888 & 0.14311 & 82.3 & 1.306 & -0.0 & 1.235 & 0.0 & 1.589 & 0.4 \\
\hline $3 f_{0}+f_{m}$ & 8.355238 & 0.00336 & 105.0 & 1.381 & -10.7 & 1.091 & 2.7 & 1.965 & 19.5 \\
\hline $4 f_{0}-f_{m}$ & 10.700834 & 0.00723 & 150.7 & 1.313 & -3.7 & 1.300 & -4.4 & 1.449 & -4.0 \\
\hline $4 f_{0}$ & 10.889184 & 0.09797 & 42.6 & 1.300 & 0.1 & 1.226 & -0.6 & 1.577 & -1.0 \\
\hline $4 f_{0}+f_{m}$ & 11.077534 & 0.00382 & 81.3 & 1.264 & 8.1 & 1.492 & -19.9 & 2.403 & 7.3 \\
\hline $5 f_{0}-f_{m}$ & 13.423130 & 0.00590 & 105.4 & 1.164 & -2.2 & 1.143 & -0.4 & 1.827 & 2.4 \\
\hline $5 f_{0}$ & 13.611480 & 0.05957 & 7.7 & 1.278 & 0.6 & 1.251 & 0.2 & 1.584 & 0.8 \\
\hline $5 f_{0}+f_{m}$ & 13.799830 & 0.00249 & 41.6 & 1.100 & -0.9 & 0.984 & 1.5 & 1.705 & -4.9 \\
\hline $6 f_{0}-f_{m}$ & 16.145426 & 0.00559 & 64.5 & 1.170 & 1.9 & 1.337 & -3.9 & 1.775 & -2.5 \\
\hline $6 f_{0}$ & 16.333776 & 0.04308 & 324.0 & 1.294 & -0.9 & 1.240 & -0.4 & 1.594 & -0.5 \\
\hline $6 f_{0}+f_{m}$ & 16.522126 & 0.00256 & 348.2 & 1.371 & 15.2 & 1.882 & -3.3 & 2.048 & 12.3 \\
\hline $7 f_{0}-f_{m}$ & 18.867722 & 0.00462 & 36.4 & 1.409 & -0.1 & 1.467 & 5.6 & 1.668 & 10.7 \\
\hline $7 f_{0}$ & 19.056072 & 0.03283 & 287.5 & 1.263 & -1.3 & 1.243 & 2.1 & 1.636 & 0.3 \\
\hline $7 f_{0}+f_{m}$ & 19.244422 & 0.00172 & 336.9 & 1.320 & -26.5 & 1.117 & -1.0 & 1.564 & 11.9 \\
\hline $8 f_{0}-f_{m}$ & 21.590018 & 0.00364 & 355.7 & 1.275 & -5.0 & 1.071 & 8.2 & 2.011 & 13.9 \\
\hline $8 f_{0}$ & 21.778368 & 0.02139 & 248.1 & 1.262 & 0.2 & 1.280 & 2.4 & 1.695 & 1.4 \\
\hline $8 f_{0}+f_{m}$ & 21.966718 & 0.00102 & 261.8 & 1.539 & 4.9 & 1.457 & 21.5 & 1.172 & 2.7 \\
\hline $9 f_{0}-f_{m}$ & 24.312314 & 0.00278 & 308.1 & 1.068 & -4.2 & 1.187 & 6.3 & 2.122 & -1.0 \\
\hline $9 f_{0}$ & 24.500664 & 0.01513 & 198.3 & 1.294 & 0.7 & 1.192 & 0.2 & 1.589 & -1.8 \\
\hline $9 f_{0}+f_{m}$ & 24.689014 & 0.00162 & 231.2 & 1.562 & 20.7 & 1.514 & -11.1 & 1.421 & -11.7 \\
\hline $10 f_{0}-f_{m}$ & 27.034610 & 0.00200 & 266.9 & 1.305 & -14.7 & 1.250 & 2.6 & 1.563 & 21.1 \\
\hline $10 f_{0}$ & 27.222960 & 0.01126 & 162.4 & 1.283 & -4.7 & 1.169 & 1.9 & 1.532 & 2.8 \\
\hline $11 f_{0}-f_{m}$ & 29.756906 & 0.00135 & 206.1 & 1.689 & 13.6 & 0.849 & -14.5 & 1.262 & -8.4 \\
\hline $11 f_{0}$ & 29.945256 & 0.00842 & 114.7 & 1.290 & -7.5 & 1.168 & -0.1 & 1.815 & 9.8 \\
\hline $12 f_{0}-f_{m}$ & 32.479202 & 0.00167 & 185.4 & 1.275 & -1.5 & 0.898 & 5.9 & 1.653 & 23.0 \\
\hline $12 f_{0}$ & 32.667552 & 0.00621 & 64.6 & 1.417 & 1.3 & 1.250 & 2.7 & 1.451 & 1.3 \\
\hline $13 f_{0}-f_{m}$ & 35.201498 & 0.00105 & 127.3 & 1.486 & 0.2 & 0.938 & 1.1 & 1.522 & 33.6 \\
\hline $13 f_{0}$ & 35.389848 & 0.00534 & 19.8 & 1.356 & -3.1 & 1.236 & 0.6 & 1.745 & 1.2 \\
\hline $14 f_{0}-f_{m}$ & 37.923794 & 0.00121 & 87.1 & 1.438 & 19.9 & 1.322 & -13.1 & 1.080 & -2.4 \\
\hline $14 f_{0}$ & 38.112144 & 0.00402 & 332.4 & 1.313 & -4.0 & 1.371 & 6.6 & 1.558 & 6.8 \\
\hline $15 f_{0}-f_{m}$ & 40.646090 & 0.00162 & 56.1 & 1.117 & -10.2 & 1.443 & 10.7 & 2.492 & 19.4 \\
\hline $15 f_{0}$ & 40.834440 & 0.00351 & 281.7 & 1.291 & 0.7 & 1.167 & 10.2 & 1.469 & 3.6 \\
\hline $16 f_{0}$ & 43.556736 & 0.00333 & 243.1 & 1.300 & -10.7 & 1.140 & 6.3 & 1.617 & 13.8 \\
\hline $17 f_{0}$ & 46.279032 & 0.00281 & 181.8 & 1.580 & 6.5 & 1.373 & 3.5 & 1.813 & 21.6 \\
\hline $18 f_{0}$ & 49.001328 & 0.00250 & 146.9 & 1.488 & -2.1 & 1.471 & -3.0 & 1.736 & 11.6 \\
\hline $19 f_{0}$ & 51.723624 & 0.00192 & 96.7 & 1.651 & 12.3 & 0.905 & -1.3 & 1.049 & 15.0 \\
\hline $20 f_{0}$ & 54.445920 & 0.00280 & 55.8 & 1.168 & 7.0 & 1.544 & 3.5 & 1.818 & 20.8 \\
\hline $21 f_{0}$ & 57.168216 & 0.00221 & 19.3 & 0.986 & 3.3 & 1.435 & 14.7 & 2.105 & 20.3 \\
\hline
\end{tabular}


Table 1-Continued

\begin{tabular}{rrrrrrrrrr}
\hline \hline & frequency c/d & \multicolumn{1}{c}{$A_{V} \operatorname{mag}$} & $\phi_{V}$ deg. & \multicolumn{1}{c}{$A_{B} / A_{V}$} & $\phi_{B}-\phi_{V}$ & $A_{V} / A_{R}$ & $\phi_{V}-\phi_{R}$ & $A_{V} / A_{I}$ & $\phi_{V}-\phi_{I}$ \\
\hline $22 f_{0}$ & 59.890512 & 0.00158 & 324.1 & 1.994 & 5.6 & 1.047 & 8.3 & 1.975 & 26.9 \\
$23 f_{0}$ & 62.612808 & 0.00181 & 288.1 & 1.039 & 1.1 & 1.275 & -10.4 & 1.926 & 35.4 \\
$24 f_{0}$ & 65.335104 & 0.00206 & 263.4 & 1.087 & 0.1 & 1.537 & 10.5 & 2.512 & 8.9 \\
$25 f_{0}$ & 68.057400 & 0.00134 & 213.7 & 1.224 & 7.8 & 1.164 & 24.3 & 1.207 & 19.1 \\
\hline
\end{tabular}


Table 2. Amplitude ratios and phase differences of the modulation $\left(f_{m}\right)$, pulsation $\left(k f_{0}\right)$, and modulation 'side lobe' frequencies $\left(k f_{0} \pm f_{m}\right)$ of the different color light curve solutions for SS Cnc and RR Gem.

\begin{tabular}{|c|c|c|c|c|c|c|c|c|c|c|c|c|}
\hline Star & $A_{B} / A_{V}$ & s.dev. & $\phi_{B}-\phi_{V}$ & s.dev. & $A_{V} / A_{R}$ & s.dev. & $\phi_{V}-\phi_{R}$ & s.dev. & $A_{V} / A_{I}$ & s.dev. & $\phi_{V}-\phi_{I}$ & s.dev \\
\hline \multicolumn{13}{|l|}{$f_{m}$} \\
\hline SS Cnc & 1.15 & & -11.0 & & 1.54 & & 2.9 & & 2.46 & & 6.3 & \\
\hline RR Gem & 1.29 & & -9.8 & & 1.13 & & 9.8 & & 1.07 & & 1.6 & \\
\hline \multicolumn{13}{|l|}{$k f_{0}{ }^{\mathrm{a}}$} \\
\hline SS Cnc & 1.30 & 0.03 & -0.9 & 2.8 & 1.23 & 0.04 & 0.9 & 1.3 & 1.63 & 0.08 & 2.1 & 3.8 \\
\hline RR Gem & 1.32 & 0.07 & -0.7 & 1.8 & 1.27 & 0.07 & 0.5 & 1.9 & 1.67 & 0.17 & 1.9 & 3.6 \\
\hline \multicolumn{13}{|l|}{$k f_{0} \pm f_{m}^{\mathrm{a}}$} \\
\hline SS Cnc & 1.29 & 0.08 & -2.6 & 3.6 & 1.28 & 0.02 & -0.7 & 4.5 & 1.61 & 0.15 & -3.2 & 5.5 \\
\hline RR Gem & 1.36 & 0.07 & -1.1 & 4.1 & 1.22 & 0.13 & -2.5 & 7.1 & 1.55 & 0.11 & -7.7 & 6.7 \\
\hline
\end{tabular}

${ }^{a}$ Mean values and standard deviations of the amplitude ratios and phase differences of those pulsation and modulation side lobe frequency components that have $V$ amplitudes larger than or nearly equal to the amplitude of $f_{m}$ are given. This means 11 and 12 pulsation and 4 and 6 modulation frequency components for SS Cnc and RR Gem, respectively. 